\title{
A NOTE ON EXTRANEOUS INFORMATION IN REGRESSION
}

\author{
Richard BROOK and T.D. WALLACE \\ North Carolina State University, Raleigh, N.C., U.S.A.
}

The Theil--Goldberger (1961) exposition of combining sample and prior information is well known and appears now in standard textbooks for graduate econometrics courses. Diminishing the value of the prior relative to the sample information can be easily seen to lead to least squares in the limit, given the formula for the BLU estimator. However, it is not so obvious what happens when the converse limit is examined. Therefore, the purpose of this note is to show that as the variance of the errors on the priors approaches zero, the Theil-Goldberger estimator goes to the estimator implied by exact restrictions.

To review, the set-up is

$$
\begin{array}{ll}
Y=X \beta+\epsilon, & \epsilon \sim\left(0, \sigma^{2} I\right) \\
r=R \beta+u, & u \sim\left(0, \gamma^{2} I\right)
\end{array}
$$

where $Y, X, r$ and $R$ are known, $X$ and $R$ fixed of order $T \times k$ and $m \times k$, say, where $m<k<T$ and $\epsilon$ and $u$ are uncorrelated.

It is assumed that $\gamma^{2}$ is nonstochastic and $\sigma^{2}$ is fixed, not equal to zero.

The BLU estimator is:

$$
\hat{\beta}=\left(\frac{1}{\sigma^{2}} S+\frac{1}{\gamma^{2}} R^{\prime} R\right)^{-1}\left(\frac{1}{\sigma^{2}} X^{\prime} Y+\frac{1}{\gamma^{2}} R^{\prime} r\right),
$$

where $S=X^{\prime} X$.

Clearly $\hat{\beta}$ becomes the least squares estimator as the value of the prior diminishes $\left(\gamma^{2} \rightarrow \infty\right)$.

Making use of a theorem in Rao (1965) on inversion of a sum, we can rewrite eq. (3) as 


$$
\hat{\beta}=\left[\sigma^{2} S^{-1}-\sigma^{2} S^{-1} R^{\prime}\left(\sigma^{2} R S^{-1} R^{\prime}+\gamma^{2} I^{-1} R S^{-1} \sigma^{2}\right]\left[\frac{1}{\sigma^{2}} X^{\prime} Y+\frac{1}{\gamma^{2}} R^{\prime} r\right] .\right.
$$

Multiplying out the product in (4), consider the two terms:

$$
\begin{aligned}
\frac{\sigma^{2}}{\gamma^{2}} & S^{-1} R^{\prime} r-\sigma^{2} S^{-1} R^{\prime}\left(\sigma^{2} R S^{-1} R^{\prime}+\gamma^{2} I\right)^{-1} R S^{-1} R^{\prime} r \frac{\sigma^{2}}{\gamma^{2}} \\
= & \frac{\sigma^{2}}{\gamma^{2}} S^{-1} R^{\prime}\left(\sigma^{2} R S^{-1} R^{\prime}+\gamma^{2} I\right)^{-1}\left(\sigma^{2} R S^{-1} R^{\prime}+\gamma^{2} I\right) r \\
& -\frac{\sigma^{2}}{\gamma^{2}} S^{-1} R^{\prime}\left(\sigma^{2} R S^{-1} R^{\prime}+\gamma^{2} I\right)^{-1} R S^{-1} R^{\prime} r \sigma^{2} \\
= & \frac{\sigma^{2}}{\gamma^{2}} S^{-1} R^{\prime}\left(\sigma^{2} R S^{-1} R^{\prime}+\gamma^{2} I\right)^{-1}\left[\sigma^{2} R S^{-1} R^{\prime}+\gamma^{2} I-\sigma^{2} R S^{-1} R^{\prime}\right] r \\
= & \sigma^{2} R^{\prime}\left(\sigma^{2} R S^{-1} R^{\prime}+\gamma^{2} I\right)^{-1} r,
\end{aligned}
$$

which goes to $S^{-1} R^{\prime}\left(R S^{-1} R^{\prime}\right)^{-1} r$ as $\gamma^{2} \rightarrow 0$.

Thus, as $\gamma^{2} \rightarrow 0$, the estimator becomes

$$
\beta^{*}=b-S^{-1} R^{\prime}\left(R S^{-1} R^{\prime}\right)^{-1}[R b-r]
$$

where $b$ is the least squares estimator. The reader will note that $\beta^{*}$ is the restricted least squares estimator that results when the prior information is taken to be exact (e.g., Goldberger, 1964).

\section{References}

Goldberger, A.S., 1964, Econometric Theory (John Wiley, New York) p. 257.

Rao, C.R., 1965, Linear Statistical Inference and Its Applications (John Wiley, New York) p. 29.

Theil, H. and A.S. Goldberger, 1961, On pure and mixed statistical estimation in economics, International Economic Review, Jan. 1961, 65-78. 